\title{
FIXED POINTS IN CONVEX CONES
}

\author{
NICOLAS MONOD
}

AbStract. We propose a fixed-point property for group actions on cones in topological vector spaces. In the special case of equicontinuous representations, we prove that this property always holds; this statement extends the classical Ryll-Nardzewski theorem for Banach spaces. When restricting to cones that are locally compact in the weak topology, we prove that the property holds for all distal actions, thus extending the general Ryll-Nardzewski theorem for all locally convex spaces.

Returning to arbitrary actions, the proposed fixed-point property becomes a group property, considerably stronger than amenability. Equivalent formulations are established and a number of closure properties are proved for the class of groups with the fixed-point property for cones.

\section{Contents}

1. Introduction and results

2. Proof of the conical fixed-point theorems

3. Integrals and cones

4. Translate properties

5. Smaller cones

6. First stability properties

7. Central extensions

8. Subexponential groups

9. Reiter and ratio properties

10. Further comments and questions

References

\section{INTRODUCTION AND RESULTS}

As an art, mathematics has certain fundamental techniques. In particular, the area of fixed-point theory has its specific artistry.

Sister JoAnn Louise Mark Mar75, p. iii]

Traiter la nature par le cylindre, la sphère, le cône...

Paul Cézanne, letter to Émile Bernard, 15 April 1904 Ber07. p. 617]

Received by the editors January 23, 2017 and, in revised form, February 10, 2017.

2010 Mathematics Subject Classification. Primary $47 \mathrm{H} 10$. 
1.A. About fixed points. A large part of fixed-point theory for groups, and perhaps the most widely applied, takes place in the following setting:

A group $G$ acts on a compact convex set $K \neq \varnothing$ in a topological vector space $E$.

We will agree that the action on $K$ comes from a continuous linear representation on $E$; even if we are only given a continuous affine action on $K$, a suitable $G$-space $E$ can be reconstructed (as the dual of the space of affine continuous functions on $K)$.

The following two themes are of particular interest:

(I) General fixed-point theorems without any restriction on the group $G$; this requires assumptions on $K, E$, and/or the nature of the action.

(II) Make no assumptions on $K$ or on the action. Then, always having a $G$-fixed point becomes a striking property of a group $G$.

A powerful example of (I) is the original Ryll-Nardzewski theorem RN62. Here the assumptions are that $E$ is a Banach space endowed with its weak topology and that the action is (norm-)isometric. It is because compactness is only assumed in the weak topology that this result is so much stronger than earlier theorems of Kakutani Kak38 and Hahn [Hah67; Ryll-Nardzewski further generalised his statement to distal actions in general locally convex spaces [RN67] (see also GM12] for further extensions).

As for (II) this fixed-point property (considered e.g. by Furstenberg Fur63, Def. 1.4]) turns out to be equivalent to amenability Day61, [Ric67]. Although amenability was originally introduced by von Neumann [vN29] in his study of Banach-Tarski paradoxes, it has since become a familiar concept in a number of unrelated areas. The connection to (II) is that finitely additive measures normalised to have total mass one form a compact convex set.

1.B. Compact is too restrictive. The context above is too limited for certain applications. Even in the original case of Banach-Tarski paradoxes, the key question is whether our space $\mathbf{R}^{3}$ carries a finitely additive measure defined on all subsets, invariant under isometries, and normalised for a given subset $A \subseteq \mathbf{R}^{3}$ of interest, e.g. a ball. In particular, such a measure $\mu$ would generally need to be unbounded (and infinite on large subsets). A natural invariant normalisation condition is $0<\mu(g A)<\infty$ for all isometries $g$; such measures form an invariant convex cone.

For the purpose of giving examples of paradoxes, Hausdorff Hau14] and BanachTarski BT24] could bypass this issue because they could consider instead subsets of the group of rotations; there, measures can be normalised over the entire group, thus still forming a compact set.

However, the question considered by von Neumann [vN29, p. 78] is really whether there exists a finitely additive measure on a set $X$, invariant under a group $G$, and normalised for some subset $A \subseteq X$ that need not be $X$-or indeed be invariant. In fact Tarski proved that this is equivalent to the non-paradoxicality of $A$ Tar38, end of $\S 3]$. Groups that always admit such a measure are called supramenable since Rosenblatt Ros72, 4.5].

It is known that supramenability is much stronger than amenability. It fails to hold for metabelian groups such as the Baumslag-Solitar group BS(1,2) or the lamplighter group $\mathbf{Z} / 2$ భ $\mathbf{Z}$ because supramenable groups cannot contain non-abelian 
free semigroups [Ros72, 2.3]. In fact, supramenability is equivalent to not containing a Lipschitz-embedded binary tree [KMR13, Prop. 3.4].

On the other hand, examples of supramenable groups include all abelian groups, all locally finite groups, and more generally the groups that are subexponential in the sense that for every finite subset $S$ of the group, $\lim _{n \rightarrow \infty}\left|S^{n}\right|^{1 / n}=1$; see $[$ Ros72, 3.5]. This argument was in fact already used in 1946 by Sierpiński; see pp. 115-117 in Sie46].

Another situation where compact convex sets are too restrictive is the case of Radon measures on locally compact spaces. Amenability can be characterised in terms of the existence of invariant probability measures on compact topological spaces, but the non-compact case is of course also very interesting (we think e.g. of Haar measures); it has been explored e.g. in KMR13, MR15. The positive Radon measures form, again, a convex cone; it has in general no affine base. A basic example is the affine group of the line, which is amenable but does not fix any non-zero Radon measure on the line.

Many other examples of convex cones without base arise, for instance in noncommutative operator algebras; see e.g. ERS11.

1.C. Convex cones. Our starting point will be the following, to be further refined below (while earlier work is briefly reviewed in Section 10.C):

A group $G$ acts on a convex proper cone $C \subseteq E$ in a topological vector space $E$. The question is whether there exists a non-zero fixed point.

Recall that proper means $C \cap(-C)=\{0\}$, or equivalently that the associated pre-order on $E$ is an order. It is understood that a representation of a group on a vector space $E$ is an action by linear maps that are assumed continuous (hence weakly continuous) if a topology is given on $E$ and order-preserving if a vector space pre-order (equivalently, a convex cone) is given for $E$. We then also speak of a representation on the cone, but the ambient linear representation is understood as part of the data.

The topological condition on $C$ shall be that $C$ is weakly complete. Choquet has eloquently made the case in his ICM address [Cho63 that this condition captures many familiar cones encountered in analysis. Oftentimes, the spaces $E$ that we need to consider are weak in the sense that their topology coincides with the associated weak topology; in any case, the weak completeness assumption justifies that we shall only consider locally convex Hausdorff topological vector spaces (as is generally done in the compact convex case too).

For instance, the cone of positive Radon measures on a locally compact space is complete for the vague topology (Proposition 14 in [Bou65, III, §1, No.9]), hence weakly complete since this topology is weak.

This setting obviously includes the earlier picture, for if $K \subseteq E$ is convex and compact or weakly compact, then the cone

$$
C=K \rtimes \mathbf{R}_{+}:=\{(t k, t): t \geq 0, k \in K\} \quad \text { in } E \oplus \mathbf{R}
$$

is convex, proper, and weakly complete -indeed even weakly locally compact [Bou81, II, §7, No.3]. This special case admits a base, $K \times\{1\}$, and this base is even $G$-invariant. 
1.D. How groups act on cones. Having given up compactness, we need some bounds on the actions on cones; otherwise, even the group $\mathbf{Z}$ will act without non-zero fixed point on any cone, simply by non-trivial scalar multiplication. We introduce two conditions - which both trivially hold in the above case $C=K \rtimes \mathbf{R}_{+}$.

Definition 1. A representation on a convex cone $C$ in a topological vector space is of locally bounded type if there exists an element $x \neq 0$ in $C$ whose orbit is bounded. Here bounded is understood in the sense of topological vector spaces rather than orders, that is, absorbed by any neighbourhood of zero Bou81, III, §1, No. 2].

This condition holds in all the examples that we aim at. For instance, if $G$ acts on a locally compact space $X$ and $C$ is the cone $C=\mathscr{M}_{+}(X)$ of positive Radon measures, then the $G$-orbit of any Dirac mass is bounded. Indeed, the closure of the image of $X$ under the canonical map $X \rightarrow \mathscr{M}_{+}(X)$ realises the onepoint compactification of $X$ (Proposition 13 in [Bou65, III, §1, No. 9]). Notice that typically the orbits admit 0 as an accumulation point.

In this example with $C=\mathscr{M}_{+}(X)$, the convex subcone of bounded orbits is in fact dense in $C$ Bou81, III, $\S 2$, No.4], a situation that we can often reduce to (Proposition 21 below). However, it is critical to keep in mind that even then, there are usually also unbounded orbits. If for instance we let $G=\mathbf{Z}$ act by translation on $C=\mathscr{M}_{+}(\mathbf{Z}) \cong\left(\mathbf{R}_{+}\right)^{\mathbf{Z}}$, then both the bounded and the unbounded orbits are dense; there are even invariant rays where $\mathbf{Z}$ acts unboundedly, namely exponentials.

We turn to the second condition, dual to local boundedness. As a motivation, we recall that any infinite group acts on some locally compact space without preserving any non-zero Radon measure [MR15, 4.3]. By contrast, it was shown in [KMR13 that we can characterise supramenability by restricting our attention to cocompact actions on locally compact spaces.

It is easily verified that for this specific example of Radon measures in the vague topology, the following general definition is precisely equivalent to the cocompactness of the $G$-action on the underlying space $X$.

Definition 2. A representation of a group $G$ on an ordered topological vector space $E$ is of cobounded type if there exists a continuous linear form $\varphi \in E^{\prime}$ which $G$-dominates all other $\lambda \in E^{\prime}$.

Here, an element $\varphi$ in an arbitrary (pre-)ordered vector $G$-space is said to $G$ dominate $\lambda$ if there exists $g_{1}, \ldots, g_{n} \in G$ with $\pm \lambda \leq \sum_{i} g_{i} \varphi$. The (topological) dual $E^{\prime}$ is always endowed with the corresponding dual $G$-representation and dual pre-order.

Remark 3. As a further motivation for this definition, another example of a weakly complete cone with a $G$-representation (of locally bounded type) without nonzero fixed point is the cone of positive elements in $\ell^{2}(G)$. This cone is weakly complete [Bec85, Prop. 5]. However, there is no non-zero fixed point as soon as $G$ is infinite.

Finally, we can formalise our group-theoretical property:

Definition 4. A group $G$ has the fixed-point property for cones if every $G$ representation of locally bounded cobounded type on any weakly complete proper convex cone has a non-zero fixed point. 
In light of theme (II) outlined at the beginning of this introduction, this is a strengthening of amenability. As we shall see, all subexponential groups enjoy the fixed-point property for cones (Theorem 8(3) ). On the other hand, this property implies supramenability and therefore the metabelian groups indicated above do not have the fixed-point property for cones.

1.E. Groups with the fixed-point property for cones. Just as the fixed-point property defined by Furstenberg turned out to be one of several equivalent incarnations of amenability, we shall establish a few characterisations in the conical setting. These equivalences (Theorem 7) will facilitate our further investigation of the fixed-point property.

Starting from our original motivation, we would like to obtain invariant finitely additive measures normalised for arbitrary subsets of a $G$-space $X$; the key case concerns subsets of $X=G$ itself. In fact, we can more generally consider invariant "integrals" normalised for functions rather than subsets. To this end, given a bounded function $f \geq 0$ on $G$, we denote by $\ell^{\infty}(G ; f)$ the ordered vector $G$-space of functions $G$-dominated by $f$.

Definition 5. Let $f \geq 0$ be a non-zero bounded function on $G$. An integral on $G$ normalised for $f$ is a $G$-invariant positive linear form $\mu$ on the vector space $\ell^{\infty}(G ; f)$ such that $\mu(f)=1$.

It should not be surprising that such integrals can be obtained as fixed points in cones. The existence of integrals under the stronger assumption that the group is subexponential was established in [Jen76, Cor. 3] and [Kel14, 2.16].

A simpler task would be to find a functional on the smaller space $\operatorname{Span}(G f)$ spanned by the $G$-orbit of $f$. However, even in the case of a subset $A \subseteq X$ with $f=\mathbf{1}_{A}$, it is not clear a priori that such a condition should imply supramenability. This amounts indeed to the question asked in Greenleaf's classical 1969 monograph Gre69, p. 18] (and met with skepticism in [Jen80, Remark on p. 369).

We shall give a positive answer to this question in Corollary 20 below. For now, we recall that there is a $G$-invariant positive linear form on $\operatorname{Span}(G f)$ normalised for $f$ if and only if the following translate property introduced in Ros72] holds for $f$.

Definition 6. Let $f \geq 0$ be a non-zero bounded function on $G$. Then $f$ satisfies the translate property if

$$
\sum_{i=1}^{n} t_{i} g_{i} f \geq 0 \Longrightarrow \sum_{i=1}^{n} t_{i} \geq 0 \quad\left(\forall t_{i} \in \mathbf{R}, g_{i} \in G\right)
$$

The group $G$ is said to have the translate property if this holds for all $f$.

This condition is equivalent to the existence of an integral on $\operatorname{Span}(G f)$ because it ensures at once that the linear form $\sum_{i} t_{i} g_{i} f \mapsto \sum_{i} t_{i}$ is both well-defined and positive. It has been known ([Gre69, §1], [Ros72, §1]) that such an integral can be extended to all of $\ell^{\infty}(G ; f)$ provided that $G$ is amenable. Therefore, we shall need to show that the translate property implies amenability.

Von Neumann's investigation of invariant finitely additive measures was not restricted to subsets of $G$ acting on itself, but it can readily be reduced to it. Likewise, we shall extend the translate property to abstract ordered vector spaces and consider the abstract translate property (defined in Section 4 below). Finally, in 
parallel to the finitary characterisations of amenability in terms of Reiter properties, we consider in Section 9 the $f$-Reiter property corresponding to a function $f$ on $G$.

Theorem 7. The following properties of a group $G$ are equivalent:

(1) the fixed-point property for cones,

(2) the translate property,

(3) the abstract translate property,

(4) for every non-zero bounded function $f \geq 0$ on $G$, there is an integral on $G$ normalised for $f$,

(5) $G$ satisfies the $f$-Reiter property for every $0 \neq f \in \ell^{\infty}(G)$.

We now investigate the class of all groups with the fixed-point property for cones, and its stability under basic group constructions. The case of metabelian groups without this property alerts us to the fact that group extensions will not, in general, preserve the fixed-point property for cones. In fact, we do not even know whether the Cartesian product (of two groups) preserves it. The same question is open for supramenable groups and was first asked in the 1970s ([Ros72, p. 49], [Ros74, p. 51]).

The closure under quotients is obvious, but for the other hereditary properties it will be helpful to be armed with the various characterisations of Theorem 7 , We find our proof of point (4) laborious; perhaps the reader will devise a simpler argument.

Theorem 8. The class of groups with the fixed-point property for cones is closed under:

(0) quotients,

(1) subgroups,

(2) directed unions,

(3) Cartesian products with subexponential groups,

(4) central extensions,

(5) extensions by and of finite groups.

1.F. Two conical fixed-point theorems. We now return to the first theme (I) namely fixed-point theorems without assumptions on the group $G$. Our first fixedpoint statement remains in the generality of weakly complete cones $C \subseteq E$ as above but under the assumption that the $G$-representation is equicontinuous on $E$. In that case, the local boundedness condition is automatically satisfied (if $C \neq\{0\}$ ).

In particular, the following contains the Ryll-Nardzewski theorem for isometries of Banach spaces as the special case of a cone $C=K \rtimes \mathbf{R}_{+}$.

Theorem 9. Let $G$ be a countable group with a representation of cobounded type on a weakly complete convex proper cone $C \neq\{0\}$ in a (Hausdorff) locally convex space $E$. If the representation is equicontinuous on $E$, then $G$ fixes a non-zero point in $C$.

Remark 10. The most general form of the Ryll-Nardzewski theorem considers actions that are distal on a compact convex set. This is a condition only on this subset of $E$, and moreover it is a weaker condition than equicontinuity (compare Gla87. and the references therein). In parallel to this generality, Theorem 12 below will address actions that are only assumed distal on a cone. At the opposite spectrum of generality, Remark 14 proposes a shorter proof for isometric representations on Banach spaces. 
The reader will have noticed that one inconspicuous assumption on $G$ did sneak into Theorem 9, namely that $G$ be countable. In the classical compact case, this restriction can immediately be lifted by a compactness argument. For the above statement, however, it is needed even in the most familiar case of Hilbert spaces:

Example 11. Let $V=L^{2}([0,1])$ and let $C$ be the convex proper cone of function classes that are a.e. non-negative. For the usual scalar product of $V$, the cone $C$ is self-dual. Since moreover $V=C-C$, it follows that $C$ is weakly complete; see e.g. Bec85, Prop. 5]. (As was pointed out to us by de la Harpe, a nice abstract characterisation of this cone was obtained by Sz.-Nagy [SN50].)

Further let $G$ be the group of non-singular automorphisms of the measure space $[0,1]$. The usual isometric $G$-representation on $V$ defined a.e. by

$$
(g f)(x)=\left(\frac{d g \mu}{d \mu}(x)\right)^{\frac{1}{2}} f\left(g^{-1} x\right) \quad\left(g \in G, f \in L^{2}\right),
$$

where $d g \mu / d \mu \in L^{1}([0,1])$ denotes the Radon-Nikodým derivative of $g \mu$ with respect to the Lebesgue measure $\mu$, preserves the cone $C$.

This $G$-action has no non-zero fixed point in $V$ but we claim that it is of cobounded type. In fact, we claim that the unit constant function $\mathbf{1} \in C$, viewed as an element of the dual, is $G$-dominating. Indeed let $f \in C$ be arbitrary and define $f_{1}=f \vee \mathbf{1} \geq f$ to be the maximum of $f$ and $\mathbf{1}$. It suffices to show that for some $g \in G$ we have $g^{-1}\left(\left\|f_{1}\right\| \mathbf{1}\right)=f_{1}$. The assignment $g(x)=\left\|f_{1}\right\|^{-2} \int_{0}^{x} f_{1}^{2} d \mu$ defines an increasing map $[0,1] \rightarrow[0,1]$ which yields a non-singular automorphism since $0<f_{1}<\infty$. Now $g^{-1}\left(\left\|f_{1}\right\| \mathbf{1}\right)=f_{1}$ holds by construction.

We can nonetheless remove this last hypothesis on $G$ if we change the topological assumption on the cone. In fact, the countability of $G$ is only used in Theorem 9 to establish that $C$ must be weakly locally compact - this is a consequence of equicontinuity which is in strong contrast to the general cones that we had to deal with for the group-theoretical property.

Once we consider cones that are locally compact in the weak topology, we can dispense of any restriction on $G$ and deal with the distal case. In particular, the following subsumes the general case of the Ryll-Nardzewski theorem as the particular instance of a cone $C=K \rtimes \mathbf{R}_{+}$.

Theorem 12. Let $G$ be a group with a representation of locally bounded type on a weakly locally compact convex proper cone $C$ in a (Hausdorff) locally convex space $E$. If the action is distal on $C$, then $G$ fixes a non-zero point in $C$.

Notice that now the coboundedness assumption has become redundant for this statement, but the local boundedness assumption came back.

For Theorem 9, the coboundedness assumption was needed even in the case of isometric actions on Banach spaces: this is illustrated by the example of $\ell^{2}(G)$ given in Remark 3 .

\section{Proof of the Conical FiXed-Point theorems}

We begin with the proof of Theorem 12 and retain its notation. One input for the proof will be established later (Proposition 21) because we shall need it again in a more general setting.

To get started, here is a consequence of the distality of the $G$-action on $C$. 
Lemma 13. Let $\epsilon>0$ and pick any $x, z \in C$ not contained in a common ray. Suppose that both orbits $G x, G z$ are bounded and that the $G$-action on $C$ is distal. Then there is a neighbourhood $U$ of the origin in $E$ such that for all $g \in G$ and all $s, t \geq \epsilon$ we have $g(s x-t z) \notin U$.

Proof. Suppose for a contradiction that the conclusion does not hold. Then there are nets of elements $g_{\alpha} \in G$ and $s_{\alpha}, t_{\alpha} \geq \epsilon$ (with index $\alpha$ in some directed set) such that $g_{\alpha}\left(s_{\alpha} x-t_{\alpha} z\right)$ converges to zero. Upon replacing them by subnets and possibly exchanging $x$ with $z$, we can assume that $s_{\alpha} \geq t_{\alpha}$ holds for all $\alpha$. Appealing to a further subnet we can assume that $t_{\alpha} / s_{\alpha}$ converges to some $\tau \in[0,1]$. We shall contradict the distality assumption by showing that $g_{\alpha}(x-\tau z)$ converges to zero, recalling that $x \neq \tau z$. Indeed, write

$$
g_{\alpha}(x-\tau z)=g_{\alpha}\left(x-\left(t_{\alpha} / s_{\alpha}\right) z\right)+\left(t_{\alpha} / s_{\alpha}-\tau\right) g_{\alpha} z .
$$

The first term is $s_{\alpha}^{-1} g_{\alpha}\left(s_{\alpha} x-t_{\alpha} z\right)$, which still converges to zero since $s_{\alpha}^{-1}$ is bounded. The second term converges to zero because $g_{\alpha} z$ is bounded in $E$.

Since $C$ is weakly locally compact and proper, it admits a weakly compact base $K_{1}$; see Exercise 21 in [Bou81, II, §7] (this result is due to Dieudonné and Klee [Kle55, 2.4]). We emphasise that this base need not be invariant. This means that we are not in the classical setting, but rather in a "projective" context; the distance between these two settings can be appreciated e.g. in light of the $T y$ chonoff property recalled in Section 10.C Nevertheless, we will be able to use suitably adapted extensions of some of the known arguments in the proofs of the Ryll-Nardzewski theorem.

Applying a compactness argument to the intersections of $K_{1}$ with subcones of fixed points in $C$, we see that it suffices to find a non-zero fixed point for each countable subgroup of $G$; we can thus assume that $G$ is countable.

We claim that we can furthermore suppose that $C$ is minimal amongst all those invariant closed convex subcones of $C$ that contain a non-zero bounded orbit. This is more delicate since the set of bounded orbits is a priori not closed, but we establish this fact in the more general weakly complete setting in Proposition 21 below. We point out that here $C$ is trivially of cobounded type as assumed in Proposition 21 because it admits a base. Notice that in particular we have reduced to the case where $C$, and hence also $K_{1}$, is separable.

We now proceed to show that $K_{1}$ contains only one point whose orbit in $C$ is bounded. Since the corresponding ray will then be $G$-invariant by uniqueness, it will in fact be pointwise fixed by distality. Therefore, this will then finish the proof.

Suppose for a contradiction that $K_{1}$ contains distinct points $x, y$ with bounded orbits and denote their midpoint by $z$. In particular the orbit of $z$ is also bounded. Let $\psi \in E^{\prime}$ be the linear form corresponding to the compact base $K_{1}$; see Bou81, II, $\S 7$, No.3]. By boundedness of the chosen orbits, there is $\epsilon>0$ such that $\psi(g x)$, $\psi(g y)$, and $\psi(g z)$ are bounded by $1 / \epsilon$ for all $g \in G$. We apply Lemma 13 to both pairs $x, z$ and $y, z$ with this $\epsilon$. Since $E$ is locally convex, the lemma implies that there exists a continuous seminorm $q$ on $E$ and $\delta>0$ such that

$$
q(g(s x-t z)) \geq \delta, \quad q(g(s y-t z)) \geq \delta \quad \forall g \in G, \forall s, t \geq \epsilon .
$$

We consider the convex weakly compact set $K_{0}=K_{1}-u \subseteq \operatorname{ker}(\psi)$ corresponding to $K_{1}$ under translation by some $u \in K_{1}$. Applying to $K_{0}$ a denting theorem such as the lemma on p. 443 in [NA67, we find a continuous linear form $\lambda_{0} \in \operatorname{ker}(\psi)^{\prime}$ 
and a constant $\alpha \in \mathbf{R}$ such that the set $U_{0}=\left\{c \in K_{0}: \lambda_{0}(c)<\alpha\right\}$ is non-empty but of $q$-diameter $<\delta$. Denote by $U_{1} \subseteq K_{1}$ the corresponding set $U_{1}=U_{0}+u$, which has the same $q$-diameter.

We choose a pre-image $\lambda_{1} \in E^{\prime}$ under the map $E^{\prime} \rightarrow E^{\prime} / \mathbf{R} \psi \cong \operatorname{ker}(\psi)^{\prime}$ and define $\lambda \in E^{\prime}$ by $\lambda=\lambda_{1}-\left(\lambda_{1}(u)+\alpha\right) \psi$. Notice that we have

$$
U_{1}=K_{1} \cap\{c \in C: \lambda(c)<0\} .
$$

In particular, by minimality of $C$ in the class of closed invariant subcones containing bounded orbits, the bounded orbit $G z$ cannot remain in the closed convex subcone $\{c \in C: \lambda(c) \geq 0\}$ of $C$. We can thus choose $g \in G$ with $\lambda(g z)<0$. At least one of $\lambda(g x)$ or $\lambda(g y)$ must also be negative; we assume $\lambda(g x)<0$. We write $s=1 / \psi(g x)$ and $t=1 / \psi(g z)$, recalling that $\psi$ does not vanish on $C \backslash\{0\}$. Now $s g x=g s x$ belongs to $U_{1}$ and likewise for $g t z$. This implies $q(g(s x-t z))<\delta$, which is a contradiction since our choice guarantees $s, t \geq \epsilon$. This completes the proof of Theorem 12 .

We now turn to Theorem 9. Consider an enumeration $\left\{g_{n}\right\}_{n=1}^{\infty}$ of $G$ and let $\varphi \in E^{\prime}$ be $G$-dominating for $E^{\prime}$. We endow $E^{\prime}$ with the weak-* topology induced by $E$. By our assumption on the action, the set $\left\{g_{n} \varphi\right\}$ is equicontinuous (on $E$ ); we use throughout that equicontinuity implies in fact uniform equicontinuity for families of linear maps (Proposition 5 in Bou74, X, $\S 2$, No. 2]). Therefore, this set is relatively compact in $E^{\prime}$; see Corollary 2 in [Bou81, III, §3, No.4]). The same holds for the convex balanced hull of $\left\{g_{n} \varphi\right\}$, since the convex balanced hull of an equicontinuous set is equicontinuous. Note that we argue by equicontinuity since, in general, the closed convex hull of a compact set need not be compact (in our case it would be true if $E$ was supposed barrelled, but not in general). It follows that we can define an element $\psi \in E^{\prime}$ by $\sum_{n=1}^{\infty} 2^{-n} g_{n} \varphi$ since the sequence of partial sums is weak-* Cauchy in this convex balanced hull.

Since $\varphi$ is $G$-dominating, $\psi$ is an order-unit for $E^{\prime}$, i.e. for any $\lambda \in E^{\prime}$ there is $t \in \mathbf{R}_{+}$with $\lambda \leq t \psi$. Thus, $\psi$ is strictly positive on $C \backslash\{0\}$; we define $K_{1}$ to be the intersection of $C$ with the affine hyperplane $\psi \equiv 1$.

We claim that $K_{1}$ is weakly compact. Since $\psi$ is an order-unit, every $\lambda \in E^{\prime}$ is bounded on $K_{1}$. Thus $K_{1}$ is weakly bounded. It is also weakly complete since $C$ is so, and therefore weak compactness follows; see e.g. Cho69, 23.11].

At this point $C$ has been shown to have a weakly compact base $K_{1}$, so that it is locally compact in the weak topology. Since the representation is equicontinuous, all orbits are bounded and the action is distal. In other words, we can apply Theorem 12 to finish the proof of Theorem 9 .

Remark 14. In the special case where $E$ is a Banach space (with isometric representation), we can instead reduce ourselves to a double application of the RyllNardzewski theorem thanks to Davis-Figiel-Johnson-Pełczyński renorming DFJP74. Indeed, the intersection $L$ of $C$ with the unit ball $E_{1}$ of $E$ is weakly compact because it is weakly complete and bounded; therefore, by the Krein-Smulian theorem, so is the closed convex hull $W$ of $L \cup(-L)$, which is now symmetric. We recall the Davis-Figiel-Johnson-Pełczyński norm $\|\cdot \mid\|$ associated to $W$. Let $\|\cdot\|_{n}$ be the gauge norm associated to the set $2^{n} W+2^{-n} E_{1}$ and define the (possibly infinite) norm $\|\cdot\|=\left(\sum_{n=1}^{\infty}\|\cdot\|_{n}^{2}\right)^{1 / 2}$. Let $F \subseteq E$ be the set of $x$ with $\|x\|$ finite and endow $F$ with the norm $\|\cdot\| \|$. Then Lemma 1 of [DFJP74] states that $F$ is a 
reflexive Banach space. By construction, the inclusion map $F \rightarrow E$ is continuous and $F$ contains $W$ in its unit ball; in particular, $C \subseteq F$.

We observe that $G$ preserves $F$ and acts isometrically for its new norm since the sets $2^{n} W+2^{-n} E_{1}$ are invariant. Moreover, it is shown in Bec89, Thm. 1] that $C$ is also weakly complete for the weak uniform structure of $F$. A weak compactness argument in the reflexive space $F$ shows that $\left.\psi\right|_{F}$ is still an order-unit for $F^{\prime}$ and a Baire argument in $F^{\prime}$ implies that $\left.\psi\right|_{F}$ is actually an upper bound for a small ball in $F^{\prime}$. This implies that the closed convex hull of $\left.G \psi\right|_{F}$ does not contain zero. By reflexivity, we can apply Ryll-Nardzewski in $F^{\prime}$ and find a non-zero positive fixed point in $F^{\prime}$. This provides an invariant base for $C$ and a second application of Ryll-Nardzewski to this base yields a fixed point in $C$.

\section{INTEGRALS AND CONES}

We begin the proof of Theorem 7 by showing that the fixed-point property for cones is, as expected, sufficiently general to be applied to cones of integrals in the sense of Definition [5] In other words, we establish (1) $\Rightarrow$ (4):

Let $f \geq 0$ be a non-zero bounded function on $G$. Consider the algebraic dual $E=\ell^{\infty}(G ; f)^{*}$ endowed with the weak-* topology defined by $\ell^{\infty}(G ; f)$. The convex cone $C \subseteq E$ of positive linear forms is proper since $\ell^{\infty}(G ; f)$ is spanned by positive elements. Moreover, $C$ is weakly complete (i.e. complete) in this topology (compare Cho62] or recall that algebraic duals are weak-* complete Bou81, II, $\S 6$, No. 7]).

To justify that the $G$-action is of locally bounded type, consider an element $g \in G$ with $f(g) \neq 0$. The evaluation at $g$ is a non-zero element $x \in C$ and we claim that its $G$-orbit is bounded in $E$. Indeed, a neighbourhood basis at 0 in $E$ is given by sets $U=\left\{y:\left|y\left(f_{i}\right)\right|<\epsilon \forall i\right\}$ with $f_{1}, \ldots, f_{n} \in \ell^{\infty}(G ; f)$ and $\epsilon>0$; we have, as required, $G x \subseteq t U$ for all large enough scalars $t$, namely $t>\max _{i}\left\|f_{i}\right\|_{\infty} / \epsilon$.

For the cobounded type condition, observe that the topological dual of $E$ is $\ell^{\infty}(G ; f)$ itself [Rud91, 3.14], endowed with the finest locally convex topology ([Sch99, p. 56], Bou81, II, §6, No.1, Rem.1)]). Therefore the condition holds since by definition $\ell^{\infty}(G ; f)$ is $G$-dominated by $f$.

At this point we apply the fixed-point property and obtain a positive element $\mu \neq 0$ in $C$. Upon renormalising, it remains only to show that $\mu(f) \neq 0$. This follows from the fact that $f$ is $G$-dominating and $\mu$ is positive.

We now prove the converse implication. Let $C$ be a weakly complete proper convex cone in a (locally convex, Hausdorff) topological vector space E. Suppose that we are given a $G$-representation of locally bounded cobounded type. We can therefore choose a non-zero point $x \in C$ with bounded $G$-orbit and a $G$-dominating element $\varphi \in E^{\prime}$. To every $\lambda \in E^{\prime}$ we associate a function $\hat{\lambda}$ on $G$ by $\hat{\lambda}(g)=\lambda(g x)$. This defines a linear positive map $\lambda \mapsto \hat{\lambda}$ from $E^{\prime}$ to the space of functions on $G$ with the pointwise order. Notice that this map is $G$-equivariant for the usual $G$-actions by pre-composition; therefore its image is $G$-dominated by $\hat{\varphi}$. Moreover, since the orbit $G x$ is bounded, $\hat{\varphi}$ is a bounded function. In conclusion, we have obtained a positive linear $G$-equivariant map from $E^{\prime}$ to $\ell^{\infty}(G ; \hat{\varphi})$.

Now let $\mu$ be an integral on $G$ normalised for $\hat{\varphi}$. Composing the above map $\lambda \mapsto \hat{\lambda}$ with $\mu$, we obtain a $G$-invariant positive linear map $\check{\mu}$ on $E^{\prime}$ with $\check{\mu}(\varphi)=\mu(\hat{\varphi})=1$. A priori $\check{\mu}$ is an element of the algebraic dual $E^{\prime *}$ of $E^{\prime}$. Suppose for a contradiction that $\check{\mu}$ is not in $C$ under the canonical embedding $C \subseteq E^{* *}$. By weak completeness, 
$C$ is closed in $E^{\prime *}$ for the weak-* topology induced by $E^{\prime}$. Therefore, the HahnBanach theorem implies that there is a continuous linear form $\lambda$ on $E^{\prime *}$ with $\left.\lambda\right|_{C} \geq \alpha$ and $\lambda(\check{\mu})<\alpha$ for some $\alpha \in \mathbf{R}$. Since $C$ is a cone, we can suppose $\alpha=0$. Moreover, by $\operatorname{Rud91}$, 3.14], we have $\lambda \in E^{\prime}$ with $\lambda(\check{\mu})=\check{\mu}(\lambda)$. Now $\lambda \geq 0$ implies that $\lambda(\check{\mu})=\mu(\hat{\lambda})$ must be positive since $\mu$ is positive and $\hat{\lambda} \geq 0$; this contradiction shows that $C$ indeed contains a non-zero fixed point, namely $\check{\mu}$.

Scholium 15. The above proof of (4) $\Rightarrow$ (1) for Theorem 7 holds unchanged if we replace the assumption of cobounded type by the following weaker assumption on $\varphi$ : there exists some non-zero point $x \in C$ with bounded $G$-orbit such that $\varphi$ is $G$-dominating for the weaker pre-order on $E^{\prime}$ given by the evaluation on the orbit $G x$ (instead of the evaluation on all of $C$, which defines the dual pre-order). This minor extension of the fixed-point property for cones also follows immediately from Proposition 21 below.

\section{Translate Properties}

As we recalled in the introduction, the translate property for a bounded nonzero function $f \geq 0$ on $G$ is equivalent to the existence of an invariant integral on $\operatorname{Span}(G f)$. However, the more important question would be whether there is an invariant integral on the larger space $\ell^{\infty}(G ; f)$. For instance, when $f=\mathbf{1}_{A}$ is the characteristic function of a subset $A \subseteq G$, this stronger property is the one that is equivalent to the existence of an invariant finitely additive measure on $G$ normalised for $A$.

Unfortunately, to go from the translate property to this stronger one, it seems that it is necessary to know a priori that $G$ is amenable; see Problem on p. 18 in Gre69.

All this is exposed in Gre69, §1] and in Ros74, but perhaps a small measure of confusion has persisted. (For instance, the amenability assumption is not used in Proposition 1.3.1 of Gre69, though it is crucial in Theorem 1.3.2; in contrast, the amenability assumption is missing in Theorem 6.3 .1 of Sha04 for $(2) \Rightarrow(3)$; finally, Corollary 1.2 of Ros74 is not a consequence, but rather a prerequisite, of the result preceding it.) We shall use notably a result of Moore [Moo13] to clarify the situation in Theorem 18 below.

We begin with a generalisation of the translate property.

Definition 16. The group $G$ has the abstract translate property if the following holds.

For every $G$-representation on an ordered vector space $(V, \leq)$ and every $v \geq 0$ in $V$ for which there is a positive linear form $\delta: V \rightarrow \mathbf{R}$ with $\delta(v) \neq 0$ which is bounded on the orbit $G v$, we have

$$
\sum_{i=1}^{n} t_{i} g_{i} v \geq 0 \Longrightarrow \sum_{i=1}^{n} t_{i} \geq 0 \quad\left(\forall t_{i} \in \mathbf{R}, g_{i} \in G\right)
$$

Let us verify that the translate property is indeed a special case of the abstract translate property with $V=\ell^{\infty}(G)$. We just need to check that for any non-zero bounded function $f \geq 0$ on $G$, there is a positive linear form $\delta$ on $\ell^{\infty}(G)$ with $\delta(f) \neq 0$ and which is bounded on the orbit $G f$. Since $f \neq 0$, we can choose $g \in G$ with $f(g) \neq 0$. Then the evaluation at $g$ is a linear form $\delta$ with the desired properties. 
The same verification shows also that the abstract translate property contains the case of the translate properties for $G$-sets considered by Rosenblatt Ros72] in the context of von Neumann's questions on finitely additive measures.

Lemma 17. The abstract translate property is equivalent to the translate property.

Proof. We need to deduce the abstract translate property from the translate property. Let $V, v$, and $\delta$ be as in Definition 16. Choose any $t_{1}, \ldots, t_{n} \in \mathbf{R}$ and $g_{1}, \ldots, g_{n} \in G$ with $\sum_{i=1}^{n} t_{i} g_{i} v \geq 0$. Define a function $f$ on $G$ by $f(g)=\delta\left(g^{-1} v\right)$; observe that this definition is dual to the one made in the proof of (4) $\Rightarrow$ (11) in Section 3 . Then $f$ is non-zero, bounded, and non-negative. For any $g \in G$, we have

$$
\sum_{i=1}^{n} t_{i} g^{-1} g_{i} v=g^{-1} \sum_{i=1}^{n} t_{i} g_{i} v \geq 0 .
$$

Applying $\delta$ we find $\sum_{i=1}^{n} t_{i} \delta\left(g^{-1} g_{i} v\right) \geq 0$ which means $\sum_{i=1}^{n} t_{i} f\left(g_{i}^{-1} g\right) \geq 0$. Since this holds for all $g \in G$, we have $\sum_{i=1}^{n} t_{i} g_{i} f \geq 0$ and hence the translate property yields $\sum_{i=1}^{n} t_{i} \geq 0$ as desired.

Theorem 18. If a group satisfies the translate property, then it is amenable.

Proof. By a result of Moore, it suffices to show that for every subset $E \subseteq G$ there is a positive linear form $\mu$ on $\ell^{\infty}(G)$ normalised for $\mathbf{1}_{G}$ and such that $\mu\left(\mathbf{1}_{E}\right)=\mu\left(\mathbf{1}_{g E}\right)$ for all $g \in G$; see Theorem 1.3 in [Moo13].

Consider the subspace $D<\ell^{\infty}(G)$ spanned by all $\mathbf{1}_{E}-\mathbf{1}_{g E}$ as $g$ ranges over $G$. Denote by $Q$ the quotient space $\ell^{\infty}(G) / \bar{D}$ with the quotient norm (where $\bar{D}$ denotes the norm-closure).

We claim that the $Q$-norm of the canonical image of $\mathbf{1}_{G}$ is one. For otherwise we can find $\epsilon>0, t_{1}, \ldots, t_{n} \in \mathbf{R}$ and $g_{1}, \ldots, g_{n} \in G$ such that $v=\sum_{i} t_{i}\left(\mathbf{1}_{E}-\mathbf{1}_{g_{i} E}\right)$ satisfies

$$
\left\|\mathbf{1}_{G}-v\right\|_{\infty} \leq 1-\epsilon .
$$

Thus $\mathbf{1}_{G}-v \leq(1-\epsilon) \mathbf{1}_{G}$ and hence $v \geq \epsilon \mathbf{1}_{G}$. In particular, $v-\epsilon \mathbf{1}_{E}$ is non-negative on $G$. However, such an element $v-\epsilon \mathbf{1}_{E}$ stands in violation of the translate property because the sum of its coefficients is $-\epsilon$. This proves the claim.

Now the Hahn-Banach theorem provides a continuous linear form $\tilde{\mu}$ of norm one on $Q$ such that $\tilde{\mu}\left(\mathbf{1}_{G}+\bar{D}\right)=1$. The corresponding linear form $\mu$ on $\ell^{\infty}(G)$ satisfies $\mu\left(\mathbf{1}_{G}\right)=1$ and is still of norm one; in particular, $\mu$ is positive. Since $\mu$ vanishes on $D$, it satisfies $\mu\left(\mathbf{1}_{E}\right)=\mu\left(\mathbf{1}_{g E}\right)$ for all $g \in G$ as requested.

The following consequence of Theorem 18 completes the proof of Theorem 7. except for point (5) which we defer to Section 9 .

Corollary 19. The group $G$ satisfies the translate property if and only if for every non-zero bounded function $f \geq 0$ on $G$, there is an integral on $G$ normalised for $f$.

The proof of Theorem 18 given above uses only the fact that $G$ satisfies the translate property for characteristic functions of subsets of $G$. Therefore, we deduce the following variant of Corollary 19.

Corollary 20. A group satisfies the translate property for sets if and only if it is supramenable. 


\section{SMaller CONES}

In preparation for the proof of Theorem 8 , we begin with two reduction statements.

Proposition 21. In the definition of the fixed-point property for cones, one can assume in addition that the cone is minimal as an invariant closed convex cone containing a non-zero bounded orbit. In particular, one can assume that the bounded orbits are dense in the cone.

The reason why the minimality statement is not completely obvious is that the union of bounded orbits is generally not closed.

Proof of Proposition 21. We consider a group $G$ with a representation on a weakly complete cone $C \subseteq E$. We suppose that there is a non-zero element with a bounded $G$-orbit in $C$ and that $\varphi \in E^{\prime}$ is $G$-dominating.

For the minimality, it suffices to show that for any chain $\mathscr{C}$ of closed $G$-invariant convex cones $D \subseteq C$ containing non-zero bounded orbits, the intersection $\bigcap \mathscr{C}$ still contains a non-zero bounded orbit. Indeed, a linear form $\varphi \in E^{\prime}$ that is $G$ dominating with respect to the pre-order dual to $C$ is a fortiori dominating for the pre-order induced on $E^{\prime}$ by any subcone of $C$, and closed convex subcones are weakly closed and hence remain weakly complete.

Thus let $\mathscr{C}$ be such a chain and choose in each $D \in \mathscr{C}$ a point $x_{D} \neq 0$ whose orbit is bounded. Since $\varphi$ is $G$-dominating, there is $g \in G$ (depending on $D$ ) with $\varphi\left(g x_{D}\right)>0$. Since the orbit of $x_{D}$ is bounded, we can take $g$ such that moreover $\varphi\left(g x_{D}\right) \geq \frac{1}{2} \sup \varphi\left(G x_{D}\right)$. Therefore we can change our choice of $x_{D}$ by translating and renormalising, and assume that $\varphi\left(x_{D}\right) \geq 1 / 2$ and $\sup \varphi\left(G x_{D}\right)=1$ hold for all $D$.

Since $\varphi$ is $G$-dominating, it follows that for every $\lambda \in E^{\prime}$, the value $\lambda\left(x_{D}\right)$ is bounded independently of $D$. Therefore, the net $\left(x_{D}\right)_{D \in \mathscr{C}}$ has an accumulation point $x$ in the weak completion of $E$ and $x \in C$ by weak completeness of the cone. Since $\mathscr{C}$ is a chain of weakly closed sets, $x \in \bigcap \mathscr{C}$. Moreover, $x \neq 0$ because $\varphi(x) \geq$ $1 / 2$. Finally, the $G$-action being weakly continuous, we have $\varphi(g x) \leq 1$ for all $g \in G$; by $G$-domination, this implies that the orbit $G x$ is weakly bounded, and thus bounded by the generalised Banach-Steinhaus principle (Corollary 3 in Bou81, III, $\S 5$, No. 3] or [Rud91, 3.18]).

The density statement follows from minimality since the set of points with bounded orbit is a $G$-invariant convex cone in $C$.

A different notion of smallness is provided by the following result.

Proposition 22. For countable groups, the fixed-point property for cones holds as soon as it holds for all cones in spaces whose weak topology is first-countable.

We cannot prove this simply by replacing $C$ with the closed convex cone spanned by a bounded $G$-orbit, because weakly separable spaces or cones are usually far from weakly first-countable ( $L^{2}$ provides examples). It also seems that one cannot conduct a limit argument by exhausting $\ell^{\infty}(G ; f)$ by subspaces of countable dimension. The reason is that subspaces of countable dimension cannot, in general, be full in the sense of ordered vector spaces - a fortiori not ideals in the Riesz space $\ell^{\infty}(G ; f)$. This deprives us from the likes of the compactness argument invoked in Proposition 21. 
Proof of Proposition 22. Consider the argument given in Section 3 for the implication (1) $\Rightarrow$ (4) of Theorem 7 we retain its notation. Exactly the same argument can be applied if we replace the space $\ell^{\infty}(G ; f)$ by the subspace $\operatorname{Span}(G f)$ spanned by the $G$-orbit of $f$. This leads to the a priori weaker conclusion that there is a $G$-invariant positive linear form on $\operatorname{Span}(G f)$, normalised for $f$. This implies the translate property, which we have proved to be in fact equivalent to the existence of an integral on $G$ normalised for $f$ (Corollary 19 above). As we have already established in Section 3, this in turn implies the fixed-point property for cones.

The reason why we travel this circuitous route is that when $G$ is countable, it is sufficient in this argument to test the fixed-point property for cones only in spaces $E$ whose weak topology admits a countable neighbourhood basis at each point. Indeed, we used $E=\operatorname{Span}(G f)^{*}$ and this topology is the weak-* topology induced by $\operatorname{Span}(G f)$, which is a space of countable algebraic dimension.

\section{First Stability PRoperties}

We begin to address the stability properties of the class of groups with the fixed-point property for cones; closure under quotients is obvious. Thanks to Theorem 7 , the closure under subgroups and directed unions becomes easy. Indeed, for subgroups it suffices to show the following.

Lemma 23. Let $G$ be a group with the translate property. Then any subgroup $H<G$ has the translate property.

Proof. If $f \geq 0$ is a non-zero bounded function on $H$, we consider it as a function on $G$ by extending it by zero outside $H$. Then the translate property for $f$ with respect to $G$ implies in particular the corresponding property with $H$.

Remark 24. Denote by $f \mapsto \tilde{f}$ the extension by zero outside $H$ in $G$; this yields a positive linear $H$-equivariant map $\ell^{\infty}(H ; f) \rightarrow \ell^{\infty}(G ; \tilde{f})$. This shows that one can also establish the closure under subgroups using the characterisation in terms of integrals.

As for directed unions, we only need the following.

Lemma 25. Suppose that the group $G$ is the union of a family $\left\{G_{\alpha}\right\}_{\alpha}$ of subgroups which is directed with respect to inclusion. If each $G_{\alpha}$ has the abstract translate property, then $G$ has the translate property.

Proof. Suppose that we have a counterexample to the translate property for $G$, namely $f \geq 0$ in $\ell^{\infty}(G), t_{1}, \ldots, t_{n} \in \mathbf{R}$, and $g_{1}, \ldots, g_{n} \in G$ with $\sum_{i} t_{i} g_{i} f \geq 0$ but $\sum_{i} t_{i}<0$. Then this violates the abstract translation property for any $G_{\alpha}$ containing all $g_{i}$ considered with its representation on $\ell^{\infty}(G)$. Such a $G_{\alpha}$ exists since the union is directed.

As we have already mentioned, group extensions do not preserve the fixed-point property for cones. However, extensions by and of finite groups are covered by the next two lemmas.

Lemma 26. Let $G$ be a group and $H<G$ a finite index subgroup. If $H$ has the fixed-point property for cones, then so does $G$.

Proof. Consider a $G$-representation on a weakly complete convex proper cone $C \subseteq$ $E$ of locally bounded cobounded type. In order to apply the fixed-point property 
of $H$, we only need to justify that the $H$-action is of cobounded type. Thus let $\varphi \in E^{\prime}$ be $G$-dominating. We fix a set $R \subseteq G$ of representatives for the right cosets of $H$ in $G$ and define $\varphi_{*}=\sum_{r \in R} r \varphi$. Then $\varphi_{*}$ is $H$-dominating because any finite sum $\sum_{i} g_{i} \varphi$ with $g_{i} \in G$ can be written $\sum_{i} h_{i} r_{i} \varphi$ with $h_{i} \in H$ and $r_{i} \in R$, which is bounded by $\sum_{i} h_{i} \varphi_{*}$ because $r_{i} \varphi \leq \varphi_{*}$ for all $i$. Therefore, $H$ fixes a point $x \neq 0$ in

$C$. Now $x^{*}=\sum_{r \in R} r^{-1} x$ is a $G$-fixed point in $C$ and $x^{*} \neq 0$ because $C$ is a proper cone.

Lemma 27. Let $G$ be a group and $F \triangleleft G$ a finite normal subgroup. If $G / F$ has the fixed-point property for cones, then so does $G$.

Proof. We consider again a $G$-representation on $C \subseteq E$ as above. Since the subspace $E^{F}$ of $F$-fixed points is closed, it suffices to justify that we can apply the fixed-point property to the convex proper cone $C^{F}=C \cap E^{F}$ in the $G / F$-space $E^{F}$, this cone remaining weakly complete. This time the cobounded type is automatic because the map $E^{\prime} \rightarrow\left(E^{F}\right)^{\prime}$ is equivariant, positive, and onto by Hahn-Banach. We need to check that $C^{F}$ has a non-zero point with bounded $G / F$-orbit (which is its $G$-orbit). Since $C$ is proper, the point $\sum_{g \in F} g x$ will do whenever $x \neq 0$ is a point of $C$ with bounded $G$-orbit.

\section{Central extensions}

In order to prove that any central extension of a group with the fixed-point property for cones retains this property, we can restrict ourselves to the case of countable groups thanks to points (1) and (2) of Theorem 8, Indeed, a subgroup of a central extension is a central extension of a subgroup. Our strategy is to prove that if a group $G$ has a representation of locally bounded cobounded type on a weakly complete convex proper cone $C \subseteq E$ which is minimal in the sense of Proposition 21, then the centre of $G$ acts trivially on $C$. This then establishes the stability under central extensions.

However, it will be crucial to know that we can in addition assume the weak topology of $E$ to have a countable neighbourhood basis, because this allows us to argue with extreme rays. Recall that even very familiar cones in separable Hilbert spaces need not have extreme rays (and their weak topology is not first-countable). This is the case of the self-dual cone in $L^{2}$ considered in Example 11.

In order to ensure the countable neighbourhood basis, we apply Proposition 21 to the cone provided by Proposition 22. We now choose an arbitrary element $z$ of the centre of $G$ and proceed to prove that $z$ fixes $C$ pointwise.

The set $D=\{x+z x: x \in C\}$ is a convex $G$-invariant subcone of $C$. It is moreover closed. Indeed, if any net $\left(x_{j}+z x_{j}\right)_{j}$ with $x_{j} \in C$ converges, then so does the net $\left(x_{j}\right)$ upon passing to a subnet because the sum map $C \times C \rightarrow C$ is proper; see Corollary 1 in [Bou81, II, §6, No. 8] together with Theorem 1(c) in [Bou71, I, $\S 10$, No. 2]. This implies that the limit of $\left(x_{j}+z x_{j}\right)_{j}$ belongs to $D$. Furthermore, $D$ contains a non-zero bounded orbit because the map $x \mapsto x+z x$ is $G$-equivariant. Therefore, by minimality, $D=C$.

It follows that every extreme ray of $C$ is set-wise preserved by $z$. As was already mentioned, there need not exist any extreme ray in general; however, the fact that $E$ has a countable neighbourhood basis implies that $C$ is indeed the closed convex hull of its extreme rays; see Proposition 5 in [Bou81, II, §7, No.2]. 
Thus, for $x \neq 0$ on an extreme ray, there is a positive scalar $\lambda_{x}$ such that $z x=\lambda_{x} x$. On the other hand, for any $\lambda>0$ we define closed convex $G$-invariant subcones $C_{\lambda}^{ \pm} \subseteq C$ by $C_{\lambda}^{+}=\{x \in C: z x \geq \lambda x\}$ and $C_{\lambda}^{-}=\{x \in C: z x \leq \lambda x\}$. A priori we cannot play the minimality of $C$ against $C_{\lambda}^{ \pm}$because we do not know whether these cones contain non-zero bounded $G$-orbits. But $C_{\lambda}^{+}+C_{\lambda}^{-}$is still a convex $G$-invariant subcone and it is closed by Corollary 2 in Bou81, II, $\S 6$, No. 8]. Since every extreme ray lies in at least one of $C_{\lambda}^{+}$or $C_{\lambda}^{-}$(according to whether $\lambda_{x} \geq \lambda$ or $\left.\lambda_{x} \leq \lambda\right)$, we conclude that $C_{\lambda}^{+}+C_{\lambda}^{-}$is all of $C$.

We now claim that for each $\lambda>0$, one of the two cones $C_{\lambda}^{ \pm}$contains a non-zero bounded $G$-orbit. Indeed let $x \neq 0$ be a point of $C$ with bounded $G$-orbit. Then there exists $x^{ \pm} \in C_{\lambda}^{ \pm}$with $x=x^{+}+x^{-}$. Using again the properness of the map $C \times C \rightarrow C$, we conclude readily that both $x^{ \pm}$have bounded $G$-orbits, whence the claim since at least one of $x^{ \pm}$is non-zero.

We can now invoke minimality again and deduce that for each $\lambda>0$, one of the two cones $C_{\lambda}^{ \pm}$coincides with $C$. Upon possibly replacing $z$ by $z^{-1}$, we can assume that for $\lambda=1$ we have $C_{1}^{+}=C$. For all $\lambda>1$, we must have $C_{\lambda}^{-}=C$ since otherwise $C_{\lambda}^{+}=C$, which we claim is impossible. Indeed, there is a non-zero point $x \in C$ with bounded $G$-orbit, hence in particular bounded $\langle z\rangle$-orbit. But $z^{n} x \geq \lambda^{n} x$ allows us to apply the properness of $C \times C \rightarrow C$ to $z^{n} x=\left(z^{n} x-\lambda^{n} x\right)+\lambda^{n} x$ and deduce that $\lambda^{n} x$ is bounded, contradicting $\lambda>1$.

In conclusion, $C=\bigcap_{\lambda>1} C_{\lambda}^{-}$and $C=C_{1}^{+}$, which shows that $z$ acts trivially on $C$ - as was to be proved.

Remark 28. An alternative to Proposition 22 to ensure the existence of (enough) extreme rays when $G$ is countable is to appeal to Choquet's notion of cones that are bien coiffés (approximately translated to "well-capped"). This is a weaker property than first countability and it can be established for cones associated to $\ell^{\infty}(G ; f)^{*}$ with $G$ countable. The method consists in exhausting $\ell^{\infty}(G ; f)$ by order ideals associated to translates of $f$; although these ideals are not of countable dimension, they each give rise to a cone that can be shown to be bien coiffé. This yields a projective limit of a countable system of (non-invariant) cones which remains bien coiffé by Exercise 29(b) in [Bou81, II, $\S 7$ — cf. Theorem 23 in Cho63.

Scholium 29. A variation on a subset of the arguments in the above proof of Theorem 8(4) yields the following:

Consider a $G$-representation of locally bounded cobounded type on any weakly complete proper convex cone $C$. Let $Z: E \rightarrow E$ be any continuous linear operator which commutes with $G$ and preserves $C$. If $C$ is bien coiffé, then there is a closed convex $G$-invariant subcone $C^{\prime} \subseteq C$ which contains a non-zero bounded $G$ orbit and such that $Z$ acts by scalar multiplication on $C^{\prime}$. Indeed, we first find a subcone $C^{\prime}$ that is minimal only within the smaller class of cones $D$ satisfying in addition $Z D \subseteq D$. We then argue that $(\mathrm{Id}+Z) C^{\prime}$ must be closed in $C^{\prime}$ and deduce $(\mathrm{Id}+Z) C^{\prime}=C^{\prime}$. In particular $Z$ preserves all extreme rays of $C^{\prime}$. Since $C^{\prime}$ is also bien coiffé, it is the closed convex hull of its extreme rays. An argument with subsets $C_{\lambda}^{ \pm}$as above implies that for some $\lambda \geq 0$, the operator $Z$ coincides with $\lambda \mathrm{Id}$ on all of $C^{\prime}$. At this point we see that $C^{\prime}$ is moreover minimal in the sense of Proposition 21 (regardless of $Z$ ). 


\section{SubEXPONENTIAL GROUPS}

Recall that a group $G$ is called subexponential if $\lim _{n \rightarrow \infty}\left|S^{n}\right|^{1 / n}=1$ for every finite subset $S \subseteq G$, where $S^{n}$ is the set of $n$-fold products of elements of $S$. This holds in particular for all locally finite or abelian groups.

The following property was first recorded by Jenkins, who proved moreover that it characterises subexponentiality [Jen76, Lemma 1].

Lemma 30. Suppose $G$ is subexponential. For every finite subset $S \subseteq G$ and every $\epsilon>0$ there is $\varrho \neq 0$ in $\ell^{1}(G)$ with $|s \varrho-\varrho| \leq \epsilon \varrho$ for all $s \in S$.

Proof. Choose $\delta>0$ such that $\left|e^{\delta}-1\right| \leq \epsilon$ and $\left|e^{-\delta}-1\right| \leq \epsilon$. Define the function $\varrho$ on $G$ by $\varrho(g)=e^{-\delta|g|_{S}}$, where $|g|_{S}$ is the (symmetrised) word distance associated to $S$, with the convention $\varrho(g)=0$ if $g$ is not in the subgroup generated by $S$. The desired inequality holds by construction and $\varrho$ is summable because $G$ is subexponential.

Lemma 30 can of course also be reformulated with the inequality $|\varrho s-\varrho| \leq \epsilon \varrho$; it suffices to switch left and right multiplication by replacing $S$ with $S^{-1}$ and $\varrho$ with $h \mapsto \varrho\left(h^{-1}\right)$. In fact, the function constructed in the above proof satisfies both versions of this inequality simultaneously.

The following proposition will complete the proof of the last remaining item in Theorem 8

Proposition 31. Let $G$ be a group with the abstract translate property and let $H$ be a subexponential group. Then $G \times H$ has the translate property.

Proof. Let $f \geq 0$ be a non-zero bounded function on $G \times H$ and suppose for a contradiction that we have $g_{i} \in G, h_{i} \in H$, and $t_{i} \in \mathbf{R}$ with $\sum_{i=1}^{n} t_{i} g_{i} h_{i} f \geq 0$ but $\sum_{i=1}^{n} t_{i}<0$. Then we can choose $0<\epsilon<1$ small enough to ensure that $\sum_{i=1}^{n} \tilde{t}_{i}<0$ holds for the numbers $\tilde{t}_{i} \geq t_{i}$ defined by

$$
\tilde{t}_{i}= \begin{cases}(1+\epsilon) t_{i} & \text { if } t_{i}>0 \\ (1-\epsilon) t_{i} & \text { if } t_{i} \leq 0\end{cases}
$$

We apply the right-hand version of Lemma 30 to $H$ with this $\epsilon$ and the set $S=$ $\left\{h_{1}, \ldots, h_{n}\right\}$. This yields $\varrho \neq 0$ in $\ell^{1}(H)$ with $\left|\varrho h_{i}-\varrho\right| \leq \epsilon \varrho$ for all $i$. The usual convolution of $\ell^{1}(H)$ against $\ell^{\infty}(G \times H)$ obtained by viewing $H$ as a subgroup of $G \times H$ is

$$
(\varrho f)(x)=\sum_{y \in H} \varrho(y) f\left(y^{-1} x\right) \quad(x \in G \times H) .
$$

Furthermore, $\left(\varrho h_{i}\right) f=\varrho\left(h_{i} f\right)$ holds and $\varrho f$ is still a non-zero non-negative bounded function on $G \times H$.

Since $f \geq 0$ and $(1-\epsilon) \varrho \leq \varrho h_{i} \leq(1+\epsilon) \varrho$, we deduce

$$
(1-\epsilon) \varrho f \leq \varrho h_{i} f \leq(1+\epsilon) \varrho f \quad(\forall i) .
$$

Multiplying this by $t_{i}$ and taking into account the sign of $t_{i}$, we deduce $\tilde{t}_{i} \varrho f \geq t_{i} \varrho h_{i} f$ for all $i$.

We shall now apply the abstract translate property of $G$ to the element $\varrho f$ of $\ell^{\infty}(G \times H)$ for $g_{i}$ and $\tilde{t}_{i}$. For the linear form $\delta$ required by Definition 16] we can 
take the evaluation at any point of $G \times H$ where $f$ does not vanish. Since $\varrho$ and $g_{i}$ commute, we have

$$
\sum_{i} \tilde{t}_{i} g_{i} \varrho f \geq \sum_{i} t_{i} g_{i} \varrho h_{i} f=\varrho \sum_{i} t_{i} g_{i} h_{i} f \geq 0 .
$$

This, however, contradicts $\sum_{i=1}^{n} \tilde{t}_{i}<0$.

\section{REITER AND RATIO PROPERTIES}

This section regards approximation properties characterising the existence of integrals on $\ell^{\infty}(G ; f)$ and on $\operatorname{Span}(G f)$. It is a rather direct extension of the results known in the special case $f=\mathbf{1}_{A}$ of characteristic functions of subsets.

The following definition is a generalisation to functions of the ratio property introduced in [Ros72, §5] and [Ros73, §2] for subsets.

Definition 32. Given a function $f$ on a group $G$, we say that $G$ has the $f$-ratio property if for every finite set $S \subseteq G$ and every $\epsilon>0$ there is a finitely supported function $u$ on $G$ with

$$
\|(s u) \cdot f\|_{1}<(1+\epsilon)\|u \cdot f\|_{1} \quad \forall s \in S .
$$

Here $\|\cdot\|_{1}$ denotes the $\ell^{1}$-norm and $u \cdot f$ is the pointwise product whilst $s u$ is as usual the translation of $u$ by $s$. We will only consider the case where $f$ is bounded.

Remark 33. This definition can only be satisfied if $f \neq 0$, and we have necessarily $u \neq 0$. Moreover, the definition is unchanged if we replace $f$ by $|f|$ and we can always assume $u \geq 0$.

Next, still extending Ros72, Ros73, we consider a generalisation of the group property introduced by Dieudonné [Die60, p. 284] and now called the Reiter property.

Definition 34. $G$ has the $f$-Reiter property if for every finite set $S \subseteq G$ and every $\epsilon>0$ there is a finitely supported function $u$ on $G$ with

$$
\|(s u-u) \cdot f\|_{1}<\epsilon\|u \cdot f\|_{1} \quad \forall s \in S .
$$

The same comments as in Remark 33 hold.

Just as the Reiter property for groups characterises amenability Rei68, Ch. 8, $\S 6]$, we have:

Proposition 35. Let $f \geq 0$ be a non-zero bounded function on a group $G$. Then there is an integral on $G$ normalised for $f$ if and only if $G$ has the $f$-Reiter property.

In particular, this establishes the remaining equivalence (5) of Theorem 7

Remark 36. Rosenblatt also proposes a variant where $u$ is the characteristic function of a finite set, extending Følner's theorem [Føl55, §2]. It is not clear that this should be possible in the current context where $f$ is a function rather than a subset of $G$.

It is apparent on the definitions that the $f$-Reiter property implies the $f$-ratio property; this is consistent with the following since the existence of integrals implies the translate property.

Proposition 37. Let $f \geq 0$ be a non-zero bounded function on a group $G$. Then $f$ satisfies the translate property if and only if $G$ has the $f$-ratio property. 
In conclusion, Theorem 7 shows that the fixed-point property for cones can be characterised both by the $f$-ratio property and by the $f$-Reiter property, letting $f$ range over all non-zero positive bounded functions on $G$.

Proof of Proposition 37. We follow ideas of Rosenblatt Ros72. The $f$-ratio property implies that there is an integral on $\operatorname{Span}(G f)$ by taking an accumulation point of the net

$$
\frac{\sum_{g \in G} u(g) h(g)}{\|u \cdot f\|_{1}}, \quad h \in \operatorname{Span}(G f)
$$

indexed by $(S, \epsilon)$, with $u \geq 0$ as in Definition 32. Such an accumulation point $\mu$ exists since for any fixed $h$ the above net of scalars is bounded for cofinal indices $(S, \epsilon)$ depending on $h$. For any $g$, we have $\mu(g f)=\mu(f)$ by construction; it follows that $\mu$ is indeed invariant on $\operatorname{Span}(G f)$.

For the converse, we adapt to our setting a construction attributed to Kakutani in Ros72, p. 15]. Suppose that $f$ has the translate property and fix $(S, \epsilon)$; we can assume that $S$ contains the identity. Consider the element $v=\left(s^{-1} f\right)_{s \in S}$ of $\ell^{\infty}\left(G, \mathbf{R}^{S}\right)$ and denote by $P \subseteq\left(\mathbf{R}_{+}\right)^{S}$ the closure of the convex cone generated by the image of $v$ in $\mathbf{R}^{S}$ (in Ros72, the cone was already closed). The translate property applied to $S$ (i.e. with the elements $g_{i}$ of Definition [ enumerating $S$ ) is equivalent, by Hahn-Banach, to the statement that $\mathbf{1}_{S} \in \mathbf{R}^{S}$ belongs to $P$.

Therefore, we can choose a finite set $\left\{x_{1}, \ldots, x_{m}\right\} \subseteq G$ and coefficients $c_{j} \geq 0$ such that $\left(\sum_{j=1}^{m} c_{j}\left(s^{-1} f\right)\left(x_{j}\right)\right)_{s \in S}$ is arbitrarily close to $\mathbf{1}_{S}$. Specifically, we make the choice so that for all $s \in S$ we have $\left|1-\sum_{j=1}^{m} c_{j}\left(s^{-1} f\right)\left(x_{j}\right)\right|<\epsilon /(2+\epsilon)$. Defining $u=\sum_{j} c_{j} \delta_{x_{j}}$, this reads $\left|1-\|(s u) \cdot f\|_{1}\right|<\epsilon /(2+\epsilon)$. Since $e \in S$, we deduce

$$
\|(s u) \cdot f\|_{1}<1+\frac{\epsilon}{2+\epsilon}=(1+\epsilon)\left(1-\frac{\epsilon}{2+\epsilon}\right)<(1+\epsilon)\|u \cdot f\|_{1}
$$

as in the definition of the $f$-ratio property.

Proof of Proposition 35. The $f$-Reiter property implies the existence of an integral on $\ell^{\infty}(G ; f)$ as in the above proof of Proposition 37. noting that the stronger condition in Definition 34 ensures the invariance of the accumulation point for all $h \in \ell^{\infty}(G ; f)$ rather than just $h \in \operatorname{Span}(G f)$.

The converse can be proved as in Ros73. More precisely, the existence of an integral normalised for $f$ implies that there is a net $\left(u_{\alpha}\right)$ of finitely supported functions $u_{\alpha} \geq 0$ on $G$ with $u_{\alpha} \cdot f \neq 0$ and such that

$$
\frac{\sum_{g \in G}\left(s u_{\alpha}\right)(g) h(g)-u_{\alpha}(g) h(g)}{\left\|u_{\alpha} \cdot f\right\|_{1}} \longrightarrow 0 \quad \forall h \in \ell^{\infty}(G ; f)
$$

as in Theorem 3.3 of Ros73, noting that Ros73, Prop. 2.2] is established in the necessary generality. Going from this weak convergence to the convergence in norm of Definition 34 requires an appropriate version of the Mazur trick, established in this generality in Lemma 4.8 of Ros73.

\section{Further COMments And Questions}

10.A. Topological groups. The definition of the fixed-point property for cones can be extended to topological groups simply by adding the requirement that the 
representation be (orbitally) continuous. For applications to integrals, the corresponding change is to consider those bounded functions that are right uniformly continuous. This topological generalisation can be pursued in two directions:

On the one hand, the case of locally compact groups. Here, most arguments can be adapted with suitable technical precautions. For instance, Jenkins' criterion for subexponential groups (Lemma 1 in [Jen76]) was stated and proved in this generality.

On the other hand, for more general topological groups, the corresponding equivalent characterisations are less flexible and we cannot prove the stability properties of Theorem 8 anymore. It turns out that such statements do indeed fail; this does not, of course, make the fixed-point property for cones any less intriguing for these groups.

Example 38. Consider the Polish topological group $\mathrm{Sym}_{\infty}$ of all permutations of a countable set (with the pointwise topology). We claim that $\mathrm{Sym}_{\infty}$ has the fixedpoint property for cones as a topological group, although it contains non-abelian free groups as closed subgroups.

To justify the claim, it suffices to prove that this fixed-point property is inherited from dense subgroups, since $\mathrm{Sym}_{\infty}$ contains as a dense subgroup the group of finitely supported permutations, which has the fixed-point property for cones even as an abstract group, being a directed union of finite groups. The only non-trivial point is the following. Consider an orbitally continuous representation of a topological group $G$ on a cone $C \subseteq E$ as usual and assume that it is of locally bounded cobounded type. We need to show that the action of any dense subgroup $H$ remains of cobounded type, or at least satisfies the weaker condition of Scholium 15.

Thus let $\varphi \in E^{\prime}$ be $G$-dominating and fix some non-zero point $x \in C$ with bounded $G$-orbit. Pick any $\lambda \in E^{\prime}$. For any given $0 \neq y \in C$, the cobounded type assumption for $G$ implies that there are $g_{1}, \ldots, g_{n} \in G$ such that $\lambda(y)<\sum_{i} \varphi\left(g_{i} y\right)$ (adding, if needed, some $g$ with $\varphi(g y)>0$, which exists by domination). Now the sequence $\left(g_{i}\right)$ depends a priori on $y$ because we want this strict inequality, but this allows us to choose these $g_{i}$ in $H$ by density and orbital continuity. On the other hand, for a given sequence $g_{1}, \ldots, g_{n} \in H$, this strict inequality defines a weakly open set in $C$. Therefore, by relative weak compactness of the orbit $H x$, we can concatenate finitely many such sequences to verify the condition of Scholium 15 for $H$.

The above example is analogous to the case of amenability of topological groups that are not locally compact; see [llH82, p. 489].

10.B. Relativising. Given a subgroup $H<G$ of a group $G$, we say that $G$ has the fixed-point property for cones conditionally with respect to $H$ if the following holds: for every $G$-representation of cobounded type on any weakly complete proper convex cone that admits a non-zero $H$-fixed point with bounded $G$-orbit, there is a non-zero $G$-fixed point. Thus, the condition of locally bounded type has been conditioned on $H$.

It is straightforward to check that for normal subgroups $H \triangleleft G$, this conditional property is equivalent to the fixed-point property for cones for the quotient group $G / H$. In that sense, the conditional property is the analogue of the co-amenability studied notably by Eymard Eym72. 
These conditional properties offer a non-trivial generalisation of the corresponding absolute fixed-point properties when we consider non-normal subgroups $H<G$. For instance, in the case of amenability, it was shown that co-amenability does not pass to subgroups in the natural sense (MP03, Pes03]). In the present case, the situation is different:

Proposition 39. Consider groups $K<H<G$. If $G$ has the fixed-point property for cones conditionally with respect to $K$, then so does $H$.

This proposition is a consequence of the following one thanks to Remark 24.

Proposition 40. A group $G$ has the fixed-point property for cones conditionally with respect to $H<G$ if and only if there exists an integral on $G / H$ normalised for $f$ whenever $f \geq 0$ is a non-zero bounded function on $G / H$.

The latter proposition is proved by the arguments of Section 3 without any change.

Contrariwise, the conditional property for cones lacks a feature of co-amenability, namely it is not transitive. This is due to the fact that the fixed-point property for cones is not preserved by group extensions.

The conditional property can be further extended to group actions, generalising the $G$-action on $G / H$. In that setting, we simply replace the locally bounded type assumption by the following condition for a $G$-action on a set $X$ : we assume that there is a non-zero $G$-map from $X$ to the cone whose image is bounded. The corresponding fixed-point property can again be characterised in terms of integrals on $X$ by the arguments of Section 3 .

Finally, we point out that there is a dual way to relativise fixed-point properties to subgroups, which in the case at hand leads to the following. A subgroup $H<G$ has the fixed-point property for cones relatively to $G$ if for every $G$-representation of locally bounded type and of $H$-cobounded type on any weakly complete proper convex cone, there is an $H$-fixed point.

In the classical case of amenability, this corresponds to relative amenability as studied in CM14]. This notion turns out to occur naturally in the study of Zimmeramenability of non-singular actions on measure spaces, of group $\mathrm{C}^{*}$-algebras and boundaries Oza14, as well as of invariant random subgroups BDL16].

Just as in the case of relative amenability, this second relativisation may seem empty at first sight:

Proposition 41. For abstract groups $H<G$, the fixed-point property for cones relative to $G$ is equivalent to the fixed-point property for cones of $H$.

Indeed the property for $H$ is formally stronger and the converse follows from Remark 24.

However, in the topological context, the relative property is strictly weaker since it follows from the the fixed-point property for cones of the larger group $G$; thus, Example 38 above is a case where these properties are not equivalent.

10.C. Review of some other properties. Conical fixed point properties have been considered earlier, but mostly, as far as we know, for the special case of cones with a compact base.

In that setting, Furstenberg [Fur65, §4] introduced the notion of Tychonoff groups by requiring that a ray be preserved (not necessarily pointwise fixed). Since the 
cone has a compact base $K$, this is equivalent to fixing a point in the projective action on the compact convex set $K$. This property was further studied by ConzeGuivarc'h [GC74] and Grigorchuk Gri98]. Albeit very interesting, the Tychonoff property is not relevant to the questions we investigated here (in particular the von Neumann-Tarski invariant measure problem). Indeed, already the infinite dihedral group - which is of polynomial growth - fails to be Tychonoff. This is witnessed e.g. by its representation as a matrix group generated by $\left(\begin{array}{ll}2 & 0 \\ 0 & 1 / 2\end{array}\right)$ and $\left(\begin{array}{ll}0 & 1 \\ 1 & 0\end{array}\right)$ acting on the positive quadrant of $\mathbf{R}^{2}$. Conversely, but in the locally compact generality, connected groups of upper-triangular matrices are Tychonoff [Fur65, p. 284], but fail to be supramenable unless they have polynomial growth Jen73.

Still for cones with a compact base, Grigorchuk [Gri98, §5] considered the nonzero fixed-point property under the assumption that (all) orbits be bounded - this property follows from the fixed-point property introduced in the present article. He proved that Liouville groups enjoy that property for cones with compact bases, if we define "Liouville" by the triviality of the Poisson boundary of some finitely supported generating symmetric random walk. Liouville groups include all finitely generated subexponential groups by a result of Avez Ave74, and we can reduce to the finitely generated case by a compactness argument. But Liouville groups also include some groups with free non-abelian subsemigroups, for instance the lamplighter $\mathbf{Z} / 2$ २ $\mathbf{Z}$ (see e.g. Theorem 1.3 in Kall83); such groups have paradoxical subsets.

Jenkins has introduced in Jen80 a non-zero fixed-point property for certain compact convex sets that are allowed to be more complicated than initial slices of cones with a compact base. He proved that his fixed-point property implies the existence of integrals (Proposition 5 in [Jen80]). However, it does not hold for all subexponential groups (Remark on p. 370 in [Jen80]) and therefore is too restrictive for our purposes.

Finally, another property introduced earlier by Jenkins Jen76 is his "property F" (not to be confused with a different "property F" that he introduced earlier yet [Jen74]). We recall his definition, which is related to our abstract translate property:

Suppose that $G$ has a linear representation on a vector space $V$ and that we are given $x \in V$ and $\varphi \in V^{*}$ (the algebraic dual) such that the function $g \mapsto \varphi(g x)$ is non-zero, bounded, and $\geq 0$ on $G$. Then there exists a net $\left(\varphi_{j}\right)_{j}$ of finite positive linear combinations of $G$-translates of $\varphi$ such that, for any given $g \in G$, the net $\left(\varphi_{j}(g x)\right)_{j}$ converges to 1 .

Jenkins proved [Jen76, Thm. 2] that subexponential groups enjoy this property F. We claim that in fact this property is equivalent to our fixed-point property for cones. It is rather straightforward to see that property $\mathrm{F}$ implies the abstract translate property. Now the latter implies the fixed-point property for cones by Theorem 7 above. It remains to see that the fixed-point property for cones implies property $\mathrm{F}$. To this end, consider $\operatorname{Span}(G x)<V$ in the above notation, and endow its algebraic dual $E=\operatorname{Span}(G x)^{*}$ with the weak-* topology. Consider the cone of linear forms that are $\geq 0$ on the whole orbit $G x$. Then an argument entirely similar to that of Section 3 implies the existence of a net as required by property $\mathrm{F}$.

This equivalence also clarifies the picture with respect to another result of Jenkins: he proved [Jen76, Cor. 3] that subexponential groups, in addition to having property $\mathrm{F}$, also admit integrals. It follows from the above equivalence (and again Theorem 7) that the existence of integrals is after all equivalent to property $\mathrm{F}$. 
10.D. Questions and problems. As we recalled in the introduction, amenability is equivalent to the fixed-point property for general convex compact sets in arbitrary (Hausdorff) locally convex spaces. However, when the group is countable, it suffices to consider Hilbert spaces (in their weak topology) instead of general locally convex spaces; this was recently proved in GM17.

Question 42. Is it sufficient to check the conical fixed-point property on weakly complete cones in Hilbert spaces? How about weakly locally compact cones in Hilbert spaces?

The corresponding representations would of course not be isometric in view of Theorem 9 (for countable groups). Also, the second question is not suited for general (uncountable) groups: for instance, a group with Bergman's property Ber06. would necessarily act equicontinuously (compare GM17) and hence would have a non-zero fixed point by Theorem 12, although it might not even be amenable; an example is $\mathrm{Sym}_{\infty}$ viewed as a group without topology.

It would be desirable to have a wider menagerie of examples of groups distinguishing the properties that we consider. First in line is the following:

Problem 43. Find a finitely generated group of exponential growth enjoying the fixed-point property for cones.

In view of Theorem 8, such an example would in particular be provided by solving another problem:

Problem 44. Exhibit two groups with the fixed-point property for cones such that their product does not have this property.

We recall that the corresponding problem for supramenable groups is also open; in view of [KMR13, 3.4], the latter problem is equivalent to:

Problem 45. Give an example of two groups $G_{1}, G_{2}$ such that $G_{1} \times G_{2}$ admits a Lipschitz embedding of the binary tree but neither of the $G_{i}$ does.

Comparing the last two problems, we record that we do not know if the implication from the fixed-point property for cones to supramenability can be reversed:

Question 46. Is supramenability equivalent to the fixed-point property for cones?

The classical Reiter property has equivalent $L^{p}$ variants (already introduced in [Die60, p. 284]). Likewise, the group $G$ satisfies the $f$-ratio property for all nonzero $f \in \ell^{\infty}(G)$ if and only if the same holds with the $\ell^{1}$-norm replaced by any $\ell^{p}$-norm with $1 \leq p<\infty$. This is a straightforward verification where $f, u$, and $\epsilon$ are to be replaced with appropriate powers. The same is true for the $f$-Reiter property using basic $L^{p}$ inequalities.

The particular case of $p=2$ suggests the following:

We say that a unitary representation of a group $G$ on a Hilbert space $V$ has the operator $\alpha$-ratio property for a continuous operator $\alpha \neq 0$ of $V$ if for every finite set $S \subseteq G$ and every $\epsilon>0$, there is $v \in V$ with $\|\alpha(s v)\|<(1+\epsilon)\|\alpha(v)\|$ for all $s \in S$. Likewise, the representation has the stronger operator $\alpha$-Reiter property if we can choose $v$ with $\|\alpha(s v-v)\|<\epsilon\|\alpha(v)\|$ for all $s \in S$.

Problem 47. Clarify under which circumstances the operator $\alpha$-ratio and $\alpha$-Reiter properties hold. 
Returning closer to supramenability:

Problem 48. Characterise the unitary representations that satisfy the operator $\alpha$-ratio or $\alpha$-Reiter property for all non-zero projections $\alpha$ in $V$.

\section{ACKNOWLEDGMENTS}

The author is grateful to Taka Ozawa and to the anonymous referee for helpul remarks on preliminary versions of this article.

\section{REFERENCES}

[Ave74] André Avez, Théorème de Choquet-Deny pour les groupes à croissance non exponentielle (French), C. R. Acad. Sci. Paris Sér. A 279 (1974), 25-28. MR0353405

[BDL16] Uri Bader, Bruno Duchesne, and Jean Lécureux, Amenable invariant random subgroups, Israel J. Math. 213 (2016), no. 1, 399-422, DOI 10.1007/s11856-016-1324-7. With an appendix by Phillip Wesolek. MR3509477

[BT24] Stephan Banach and Alfred Tarski, Sur la décomposition des ensembles de points en parties respectivement congruentes, Fund. Math. 6 (1924), 244-277.

[Bec85] Richard Becker, Mesures coniques sur un espace de Banach ou son dual, J. Austral. Math. Soc. Ser. A 39 (1985), no. 1, 39-50. MR786974

[Bec89] Richard Becker, Sur les cônes faiblement complets, contenus dans un espace de Banach, Math. Ann. 285 (1989), no. 1, 99-103, DOI 10.1007/BF01442674. MR.1010193

[Ber06] George M. Bergman, Generating infinite symmetric groups, Bull. London Math. Soc. 38 (2006), no. 3, 429-440, DOI 10.1112/S0024609305018308. MR2239037

[Ber07] Émile Bernard, Souvenirs sur Paul Cézanne et lettres inédites (suite), Mercure de France 15 octobre (1907), 606-627.

[Bou65] N. Bourbaki, Éléments de mathématique. Fasc. XIII. Livre VI: Intégration. Chapitres 1, 2, 3 et 4: Inégalités de convexité, Espaces de Riesz, Mesures sur les espaces localement compacts, Prolongement d'une mesure, Espaces $L^{p}$, Deuxième édition revue et augmentée. Actualités Scientifiques et Industrielles, No. 1175, Hermann, Paris, 1965. MR 0219684

[Bou71] N. Bourbaki, Éléments de mathématique. Topologie générale. Chapitres 1 à 4, Hermann, Paris, 1971. MR0358652

[Bou74] N. Bourbaki, Éléments de mathématique. Topologie générale. Chapitres 5 à 10, Hermann, Paris, 1974.

[Bou81] N. Bourbaki, Éléments de mathématique. Espaces vectoriels topologiques. Chapitres 1 à 5, New edition, Masson, Paris, 1981. MR633754

[CM14] Pierre-Emmanuel Caprace and Nicolas Monod, Relative amenability, Groups Geom. Dyn. 8 (2014), no. 3, 747-774, DOI 10.4171/GGD/246. MR.3267522

[Cho62] Gustave Choquet, Ensembles et cônes convexes faiblement complets, C. R. Acad. Sci. Paris 254 (1962), 1908-1910. MR0132373

[Cho63] Gustave Choquet, Les cônes convexes faiblement complets dans l'analyse, Proc. Internat. Congr. Mathematicians (Stockholm, 1962), Inst. Mittag-Leffler, Djursholm, 1963, pp. 317-330. MR0179571

[Cho69] Gustave Choquet, Lectures on analysis. Vol. II: Representation theory, Edited by J. Marsden, T. Lance and S. Gelbart, W. A. Benjamin, Inc., New York-Amsterdam, 1969. MR.0250012

[DFJP74] W. J. Davis, T. Figiel, W. B. Johnson, and A. Pełczyński, Factoring weakly compact operators, J. Functional Analysis 17 (1974), 311-327. MR.0355536

[Day61] Mahlon M. Day, Fixed-point theorems for compact convex sets, Illinois J. Math. 5 (1961), 585-590. MR0138100

[Die60] Jean Dieudonné, Sur le produit de composition. II, J. Math. Pures Appl. (9) 39 (1960), 275-292. MR0115100

[ERS11] George A. Elliott, Leonel Robert, and Luis Santiago, The cone of lower semicontinuous traces on a $C^{*}$-algebra, Amer. J. Math. 133 (2011), no. 4, 969-1005, DOI 10.1353/ajm.2011.0027. MR2823868 
[Eym72] Pierre Eymard, Moyennes invariantes et représentations unitaires, Lecture Notes in Mathematics, Vol. 300, Springer-Verlag, Berlin-New York, 1972. MR0447969

[Fø155] Erling Følner, On groups with full Banach mean value, Math. Scand. 3 (1955), 243-254, DOI 10.7146/math.scand.a-10442. MR0079220

[Fur63] Harry Furstenberg, A Poisson formula for semi-simple Lie groups, Ann. of Math. (2) 77 (1963), 335-386, DOI 10.2307/1970220. MR0146298

[Fur65] Harry Furstenberg, Translation-invariant cones of functions on semi-simple Lie groups, Bull. Amer. Math. Soc. 71 (1965), 271-326, DOI 10.1090/S0002-9904-1965-11283-6. MR0177062

[GM17] Maxime Gheysens and Nicolas Monod, Fixed points for bounded orbits in Hilbert spaces, Ann. Sci. Éc. Norm. Supér. (4) 50 (2017), no. 1, 131-156, DOI 10.24033/asens.2317. MR 3621428

[GM12] Eli Glasner and Michael Megrelishvili, On fixed point theorems and nonsensitivity, Israel J. Math. 190 (2012), 289-305, DOI 10.1007/s11856-011-0192-4. MR2956242

[Gla87] Shmuel Glasner, Distal and semisimple affine flows, Amer. J. Math. 109 (1987), no. 1, 115-131, DOI 10.2307/2374554. MR878201

[Gre69] Frederick P. Greenleaf, Invariant means on topological groups and their applications, Van Nostrand Mathematical Studies, No. 16, Van Nostrand Reinhold Co., New YorkToronto, Ont.-London, 1969. MR.0251549

[Gri98] R. I. Grigorchuk, On Tychonoff groups, Geometry and cohomology in group theory (Durham, 1994), London Math. Soc. Lecture Note Ser., vol. 252, Cambridge Univ. Press, Cambridge, 1998, pp. 170-187, DOI 10.1017/CBO9780511666131.012. MR.1709958

[GC74] Y. Guivarc'h and J.-P. Conze, Propriété de droite fixe et fonctions harmoniques positives, Théorie du potentiel et analyse harmonique (Exposés des Journées de la Soc. Math. France, Inst. Recherche Math. Avancée, Strasbourg, 1973), Lecture Notes in Math., Vol. 404, Springer, Berlin, 1974, pp. 126-132. MR0399758

[Hah67] Frank Hahn, A fixed-point theorem, Math. Systems Theory 1 (1967), 55-57, DOI 10.1007/BF01692496. MR0208392

[dlH82] Pierre de la Harpe, Classical groups and classical Lie algebras of operators, Operator algebras and applications, Part I (Kingston, Ont., 1980), Proc. Sympos. Pure Math., vol. 38, Amer. Math. Soc., Providence, R.I., 1982, pp. 477-513. MR679728

[Hau14] F. Hausdorff, Bemerkung über den Inhalt von Punktmengen, Math. Ann. 75 (1914), no. 3, 428-433, DOI 10.1007/BF01563735. MR1511802

[Jen73] J. W. Jenkins, Growth of connected locally compact groups, J. Functional Analysis 12 (1973), 113-127. MR0349895

[Jen74] J. W. Jenkins, Følner's condition for exponentially bounded groups, Math. Scand. 35 (1974), 165-174, DOI 10.7146/math.scand.a-11544. MR0369609

[Jen76] J. W. Jenkins, A fixed point theorem for exponentially bounded groups, J. Functional Analysis 22 (1976), no. 4, 346-353. MR0414774

[Jen80] J. W. Jenkins, On group actions with nonzero fixed points, Pacific J. Math. 91 (1980), no. 2, 363-371. MR615684

[Ka1̆83] V. A. Kămanovich, Examples of nonabelian discrete groups with nontrivial exit boundary, Zap. Nauchn. Sem. Leningrad. Otdel. Mat. Inst. Steklov. (LOMI) 123 (1983), 167-184. MR697250

[Kak38] Shizuo Kakutani, Two fixed-point theorems concerning bicompact convex sets, Proc. Imp. Acad. 14 (1938), no. 7, 242-245. MR.1568507

[Kel14] Julian Kellerhals, Supramenable groups, Ph.D. thesis, EPFL, 2014.

[KMR13] Julian Kellerhals, Nicolas Monod, and Mikael Rørdam, Non-supramenable groups acting on locally compact spaces, Doc. Math. 18 (2013), 1597-1626. MR3158244

[Kle55] V. L. Klee Jr., Separation properties of convex cones, Proc. Amer. Math. Soc. 6 (1955), 313-318, DOI 10.2307/2032366. MR0068113

[Mar75] Joann Louise Mark, The fixed-point question for bounded non-seperating plane continua, ProQuest LLC, Ann Arbor, MI, 1975. Thesis (Educat.D.)-Oklahoma State University. MR2625850

[MR15] Hiroki Matui and Mikael Rørdam, Universal properties of group actions on locally compact spaces, J. Funct. Anal. 268 (2015), no. 12, 3601-3648, DOI 10.1016/j.jfa.2015.01.022. MR3341960 
[MP03] Nicolas Monod and Sorin Popa, On co-amenability for groups and von Neumann algebras, C. R. Math. Acad. Sci. Soc. R. Can. 25 (2003), no. 3, 82-87. MR.1999183

[Moo13] Justin Tatch Moore, Amenability and Ramsey theory, Fund. Math. 220 (2013), no. 3, 263-280, DOI 10.4064/fm220-3-6. MR3040674

[NA67] I. Namioka and E. Asplund, A geometric proof of Ryll-Nardzewski's fixed point theorem, Bull. Amer. Math. Soc. 73 (1967), 443-445, DOI 10.1090/S0002-9904-1967-11779-8. MR0209904

[Oza14] Narutaka Ozawa, Lecture on the Furstenberg boundary and $\mathrm{C}^{*}$-simplicity, Annual Meeting of Operator Theory and Operator Algebras (available online), Tokyo, 2014.

[Pes03] Vladimir Pestov, On some questions of Eymard and Bekka concerning amenability of homogeneous spaces and induced representations, C. R. Math. Acad. Sci. Soc. R. Can. 25 (2003), no. 3, 76-81. MR.1999182

[Rei68] Hans Reiter, Classical harmonic analysis and locally compact groups, Clarendon Press, Oxford, 1968. MR 0306811

[Ric67] Neil W. Rickert, Amenable groups and groups with the fixed point property, Trans. Amer. Math. Soc. 127 (1967), 221-232, DOI 10.2307/1994643. MR0222208

[Ros72] Joseph Max Rosenblatt, Invariant linear functionals and counting conditions, ProQuest LLC, Ann Arbor, MI, 1972. Thesis (Ph.D.)-University of Washington. MR2622241

[Ros73] Joseph Max Rosenblatt, A generalization of Følner's condition, Math. Scand. 33 (1973), 153-170, DOI 10.7146/math.scand.a-11481. MR0333068

[Ros74] Joseph Max Rosenblatt, Invariant measures and growth conditions, Trans. Amer. Math. Soc. 193 (1974), 33-53, DOI 10.2307/1996899. MR0342955

[Rud91] Walter Rudin, Functional analysis, 2nd ed., International Series in Pure and Applied Mathematics, McGraw-Hill, Inc., New York, 1991. MR.1157815

[RN62] Czesław Ryll-Nardzewski, Generalized random ergodic theorems and weakly almost periodic functions, Bull. Acad. Polon. Sci. Sér. Sci. Math. Astronom. Phys. 10 (1962), 271-275. MR0169984

[RN67] Czesław Ryll-Nardzewski, On fixed points of semigroups of endomorphisms of linear spaces, Proc. Fifth Berkeley Sympos. Math. Statist. and Probability (Berkeley, Calif., 1965/66), Univ. California Press, Berkeley, Calif., 1967, pp. 55-61. MR0215134

[Sch99] H. H. Schaefer and M. P. Wolff, Topological vector spaces, 2nd ed., Graduate Texts in Mathematics, vol. 3, Springer-Verlag, New York, 1999. MR 1741419

[Sha04] Yehuda Shalom, Harmonic analysis, cohomology, and the large-scale geometry of amenable groups, Acta Math. 192 (2004), no. 2, 119-185, DOI 10.1007/BF02392739. MR2096453

[Sie46] Waclaw Sierpinski, Sur la non-existence des décompositions paradoxales d'ensembles linéaires, Actas Acad. Ci. Lima 9 (1946), 113-117. MR0016442

[SN50] Béla Sz.-Nagy, Une caractérisation affine de l'ensemble des fonctions positives dans l'espace $L^{2}$, Acta Sci. Math. Szeged 12 (1950), Leopoldo Fejér et Frederico Riesz LXX annos natis dedicatus, Pars A, 228-238. MR0037467

[Tar38] Alfred Tarski, Algebraische Fassung des Maßproblems, Fund. Math. 31 (1938), 47-66.

[vN29] Johann von Neumann, Zur allgemeinen Theorie des Maßes, Fund. Math. 13 (1929), $73-116$.

EPFL, 1015 LAUsanne, SWitzerland

E-mail address: nicolas.monod@epfl.ch 